Network Working Group

Request for Comments: 4493

Category: Informational
$\mathrm{JH}$. Song

R. Poovendran

University of Washington

J. Lee Samsung Electronics

T. Iwata

Nagoya University

June 2006

The AES-CMAC Algorithm

Status of This Memo

This memo provides information for the Internet community. It does not specify an Internet standard of any kind. Distribution of this memo is unlimited.

Copyright Notice

Copyright (C) The Internet Society (2006).

Abstract

The National Institute of Standards and Technology (NIST) has recently specified the Cipher-based Message Authentication Code (CMAC), which is equivalent to the One-Key CBC MAC1 (OMAC1) submitted by Iwata and Kurosawa. This memo specifies an authentication algorithm based on CMAC with the 128-bit Advanced Encryption Standard $(A E S)$. This new authentication algorithm is named AES-CMAC. The purpose of this document is to make the AES-CMAC algorithm conveniently available to the Internet Community. 
Table of Contents

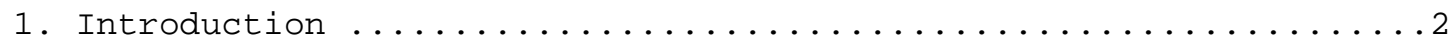

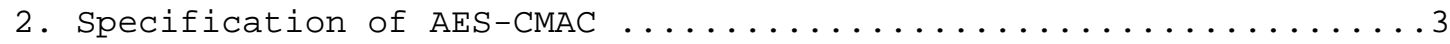

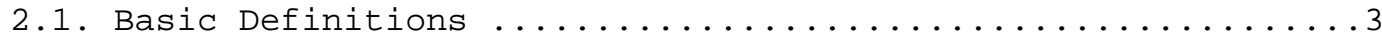

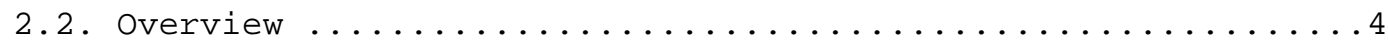

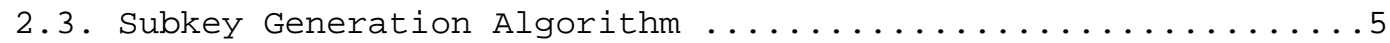

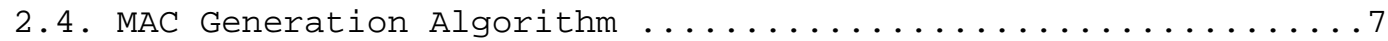

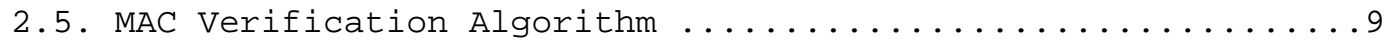

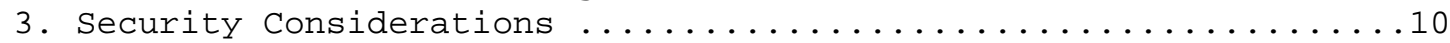

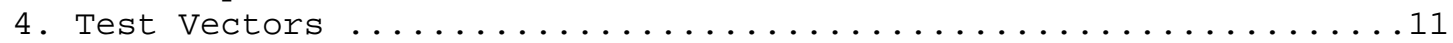

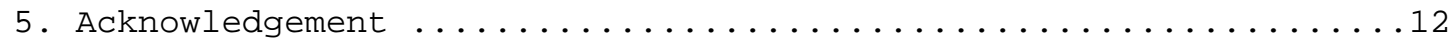

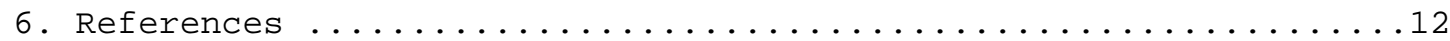

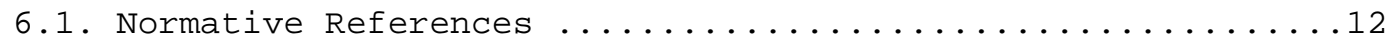

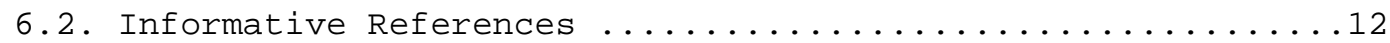

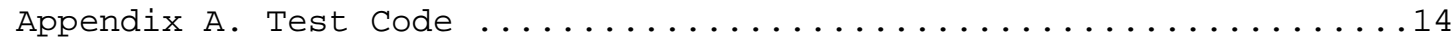

1. Introduction

The National Institute of Standards and Technology (NIST) has recently specified the Cipher-based Message Authentication Code (CMAC). CMAC [NIST-CMAC] is a keyed hash function that is based on a symmetric key block cipher, such as the Advanced Encryption Standard [NIST-AES]. CMAC is equivalent to the One-Key CBC MAC1 (OMAC1) submitted by Iwata and Kurosawa [OMAC1a, OMAC1b]. OMAC1 is an improvement of the extended Cipher Block Chaining mode (XCBC) submitted by Black and Rogaway [XCBCa, XCBCb], which itself is an improvement of the basic Cipher Block Chaining-Message Authentication Code (CBC-MAC). XCBC efficiently addresses the security deficiencies of $\mathrm{CBC}-\mathrm{MAC}$, and OMACl efficiently reduces the key size of XCBC.

AES-CMAC provides stronger assurance of data integrity than a checksum or an error-detecting code. The verification of a checksum or an error-detecting code detects only accidental modifications of the data, while CMAC is designed to detect intentional, unauthorized modifications of the data, as well as accidental modifications.

AES-CMAC achieves a security goal similar to that of HMAC [RFC-HMAC] . Since AES-CMAC is based on a symmetric key block cipher, AES, and HMAC is based on a hash function, such as SHA-1, AES-CMAC is appropriate for information systems in which AES is more readily available than a hash function.

This memo specifies the authentication algorithm based on CMAC with AES-128. This new authentication algorithm is named AES-CMAC. 


\section{Specification of AES-CMAC}

\subsection{Basic Definitions}

The following table describes the basic definitions necessary to explain the specification of AES-CMAC.

$\mathrm{x}|| \mathrm{y}$

$x$ XOR $y$

$\operatorname{ceil}(\mathrm{x})$

$\mathrm{x}<<1$

$0^{\wedge} \mathrm{n}$

$\operatorname{MSB}(\mathrm{x})$

padding (x)

Key

First subkey
Concatenation.

$\mathrm{x}|| \mathrm{y}$ is the string $\mathrm{x}$ concatenated with the string $\mathrm{y}$.

0000|| 1111 is 00001111 .

Exclusive-OR operation.

For two equal length strings, $x$ and $y$,

$\mathrm{x}$ XOR $\mathrm{y}$ is their bit-wise exclusive-OR.

Ceiling function.

The smallest integer no smaller than $x$. ceil(3.5) is 4. ceil(5) is 5 .

Left-shift of the string $x$ by 1 bit.

The most significant bit disappears, and a zero

comes into the least significant bit.

$10010001<1$ is 00100010 .

The string that consists of $\mathrm{n}$ zero-bits.

0^3 means 000 in binary format.

$10^{\wedge} 4$ means 10000 in binary format.

10^i means 1 followed by $i$-times repeated

zeros.

The most-significant bit of the string $x$. MSB (10010000) means 1 .

$10^{\wedge} i$ padded output of input $x$.

It is described in detail in section 2.4 .

128-bit (16-octet) long key for AES-128.

Denoted by $K$.

128-bit (16-octet) long first subkey, derived through the subkey

generation algorithm from the key $K$.

Denoted by $\mathrm{K} 1$. 


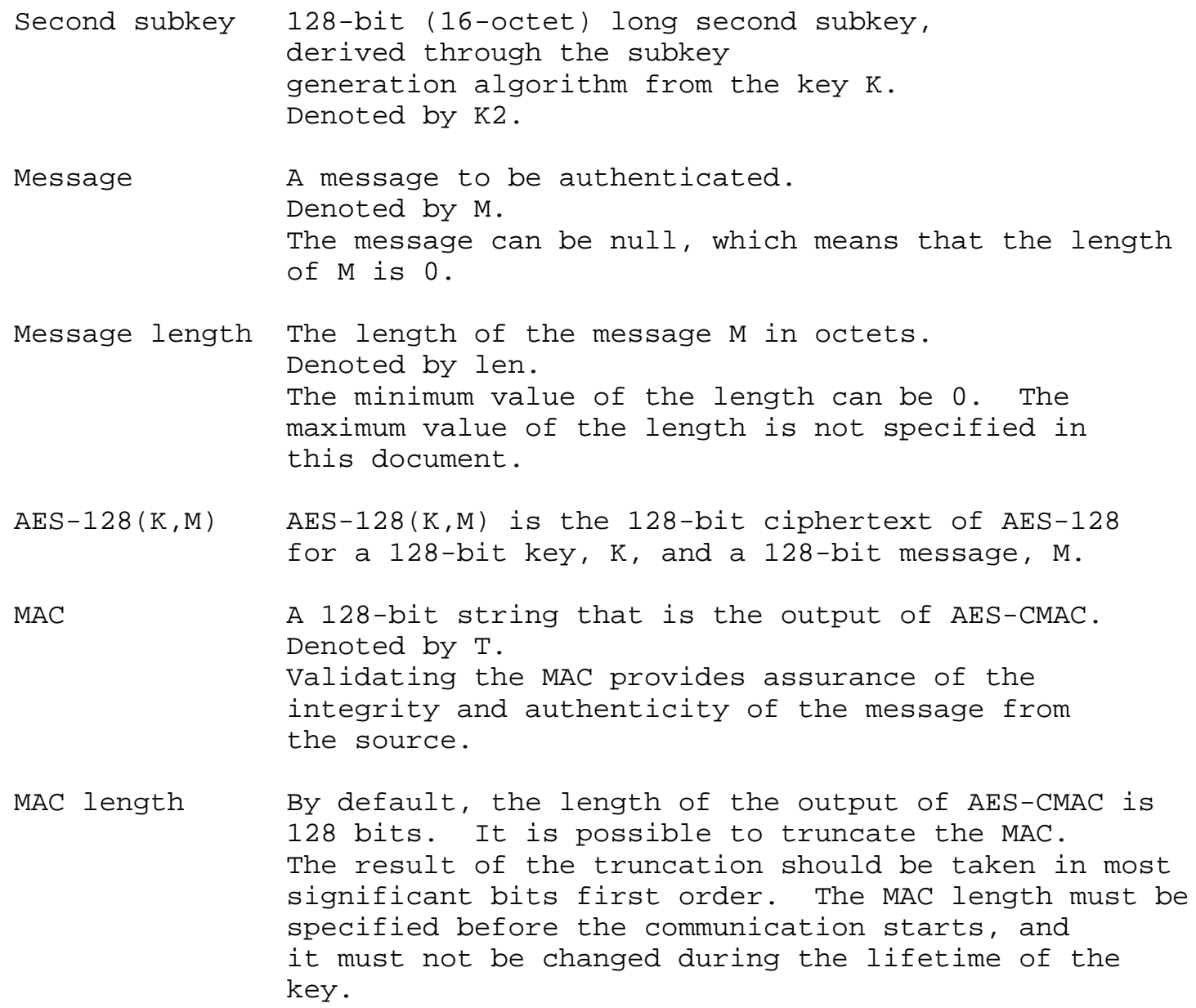

MAC length

\subsection{Overview}

AES-CMAC uses the Advanced Encryption Standard [NIST-AES] as a building block. To generate a MAC, AES-CMAC takes a secret key, a message of variable length, and the length of the message in octets as inputs and returns a fixed-bit string called a MAC.

The core of AES-CMAC is the basic CBC-MAC. For a message, M, to be authenticated, the CBC-MAC is applied to $\mathrm{M}$. There are two cases of operation in CMAC. Figure 2.1 illustrates the operation of CBC-MAC in both cases. If the size of the input message block is equal to a positive multiple of the block size (namely, 128 bits), the last block shall be exclusive-OR'ed with K1 before processing. Otherwise, the last block shall be padded with 10^i (notation is described in section 2.1) and exclusive-OR'ed with $\mathrm{K} 2$. The result of the previous 
process will be the input of the last encryption. The output of AES-CMAC provides data integrity of the whole input message.

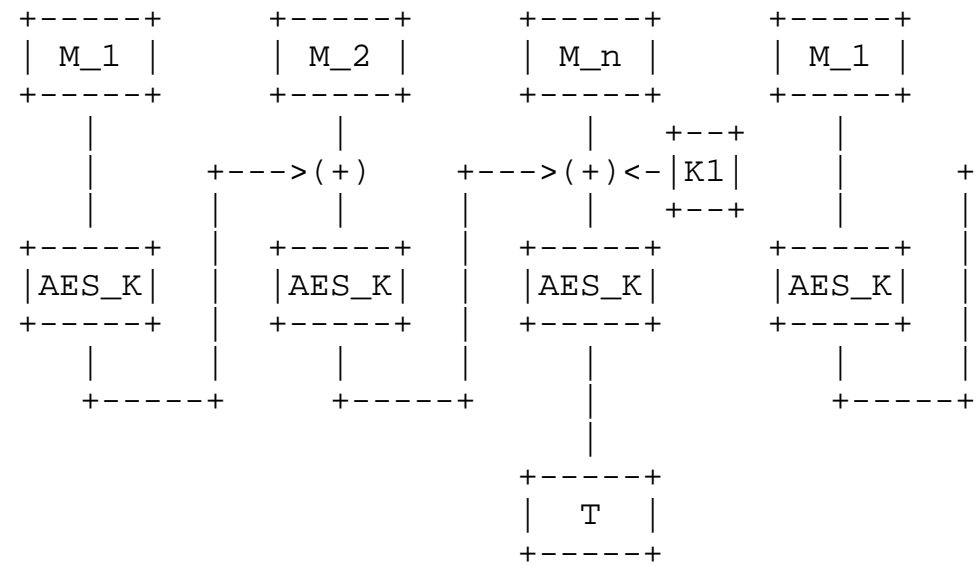

(a) positive multiple block length

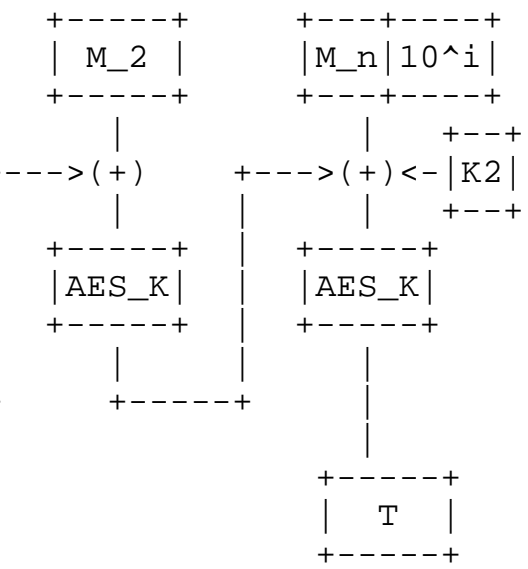

(b) otherwise

Figure 2.1. Illustration of the two cases of AES-CMAC

AES_K is AES-128 with key K.

The message $M$ is divided into blocks $M \_1, \ldots, M \_n$, where M_i is the $i$-th message block.

The length of $\mathrm{M}$ i is 128 bits for $i=1, \ldots, n-1$, and

the length of the last block, M_n, is less than or equal to 128 bits.

K1 is the subkey for the case (a), and

K2 is the subkey for the case (b).

K1 and K2 are generated by the subkey generation algorithm

described in section 2.3 .

\subsection{Subkey Generation Algorithm}

The subkey generation algorithm, Generate_Subkey(), takes a secret key, $\mathrm{K}$, which is just the key for AES-128.

The outputs of the subkey generation algorithm are two subkeys, K1 and $\mathrm{K} 2$. We write $(\mathrm{K} 1, \mathrm{~K} 2):=$ Generate_Subkey $(\mathrm{K})$.

Subkeys $\mathrm{K} 1$ and $\mathrm{K} 2$ are used in both MAC generation and MAC verification algorithms. K1 is used for the case where the length of the last block is equal to the block length. $\mathrm{K} 2$ is used for the case where the length of the last block is less than the block length. 
Figure 2.2 specifies the subkey generation algorithm.

\begin{tabular}{|c|c|c|c|}
\hline \multirow{2}{*}{$\begin{array}{l}+ \\
++\end{array}$} & \multicolumn{3}{|c|}{ Algorithm Generate_Subkey } \\
\hline & \multicolumn{3}{|c|}{+++++++++++++++++++++++++++++++++++++++++++++++++++++++++++++++++++++} \\
\hline+ & & & + \\
\hline+ & Input & : $\mathrm{K}$ (128-bit key) & + \\
\hline+ & Output & : K1 (128-bit first subkey) & + \\
\hline+ & & K2 (128-bit second subkey) & + \\
\hline \multicolumn{4}{|c|}{+} \\
\hline+ & Constants: & : const_Zero is $0 \times 00000000000000000000000000000000$ & + \\
\hline+ & & const_Rb is $0 \times 00000000000000000000000000000087$ & + \\
\hline+ & Variables: & : L for output of AES-128 applied to $0 \wedge 128$ & + \\
\hline+ & & & + \\
\hline+ & Step 1 . & $L:=\operatorname{AES}-128(\mathrm{~K}$, const_Zero $) ;$ & + \\
\hline+ & Step 2 . & if $M S B(L)$ is equal to 0 & + \\
\hline+ & & then $\quad \mathrm{K} 1:=\mathrm{L}<<1$ & + \\
\hline+ & & $\mathrm{K} 1:=(\mathrm{L}<<1)$ XOR const_Rb; & + \\
\hline+ & Step 3 . & if $M S B(K 1)$ is equal to 0 & + \\
\hline+ & & then $\quad \mathrm{K} 2:=\mathrm{K} 1<1 ;$ & + \\
\hline+ & & else $\quad \mathrm{K} 2:=(\mathrm{K} 1<1)$ XOR const_Rb; & + \\
\hline+ & Step 4 . r & return $\mathrm{K} 1, \mathrm{~K} 2$; & $\begin{array}{l}+ \\
+\end{array}$ \\
\hline
\end{tabular}

Figure 2.2. Algorithm Generate_Subkey

In step 1, AES-128 with key $\mathrm{K}$ is applied to an all-zero input block.

In step 2, K1 is derived through the following operation:

If the most significant bit of $L$ is equal to 0 , $K 1$ is the left-shift of L by 1 bit.

Otherwise, $\mathrm{K} 1$ is the exclusive-OR of const_Rb and the left-shift of $\mathrm{L}$ by 1 bit.

In step 3, K2 is derived through the following operation:

If the most significant bit of $\mathrm{K} 1$ is equal to 0 , $\mathrm{K} 2$ is the left-shift of $\mathrm{K} 1$ by 1 bit.

Otherwise, $\mathrm{K} 2$ is the exclusive-OR of const_Rb and the left-shift of K1 by 1 bit.

In step $4,(\mathrm{~K} 1, \mathrm{~K} 2):=$ Generate_Subkey $(\mathrm{K})$ is returned. 
The mathematical meaning of the procedures in steps 2 and 3 , including const_Rb, can be found in [OMACla].

\subsection{MAC Generation Algorithm}

The MAC generation algorithm, AES-CMAC(), takes three inputs, a secret key, a message, and the length of the message in octets. The secret key, denoted by $K$, is just the key for AES-128. The message and its length in octets are denoted by $\mathrm{M}$ and len, respectively. The message $M$ is denoted by the sequence of $M$ _i, where $M_{\text {_ }} i$ is the $i-t h$ message block. That is, if M consists of $\mathrm{n}$ blocks, then $\mathrm{M}$ is written as

$$
-\quad M=M \_1|| M \_2|| \ldots|| M \_\{n-1\}|| M \_n
$$

The length of $M_{-} i$ is 128 bits for $i=1, \ldots, n-1$, and the length of the last block M_n is less than or equal to 128 bits.

The output of the MAC generation algorithm is a 128-bit string, called a MAC, which is used to validate the input message. The MAC is denoted by $T$, and we write $T:=A E S-C M A C(K, M, l e n)$. Validating the MAC provides assurance of the integrity and authenticity of the message from the source.

It is possible to truncate the MAC. According to [NIST-CMAC], at least a 64-bit MAC should be used as protection against guessing attacks. The result of truncation should be taken in most significant bits first order.

The block length of AES-128 is 128 bits (16 octets). There is a special treatment if the length of the message is not a positive multiple of the block length. The special treatment is to pad $\mathrm{M}$ with the bit-string $10^{\wedge} i$ to adjust the length of the last block up to the block length.

For an input string $\mathrm{x}$ of $r$-octets, where $0<=r<16$, the padding function, padding(x), is defined as follows:

$-\operatorname{padding}(\mathrm{x})=\mathrm{x}|| 10^{\wedge} \mathrm{i} \quad$ where $i$ is $128-8 * r-1$

That is, padding $(x)$ is the concatenation of $x$ and a single '1', followed by the minimum number of ' $0^{\prime} \mathrm{s}$, so that the total length is equal to 128 bits.

Figure 2.3 describes the MAC generation algorithm. 


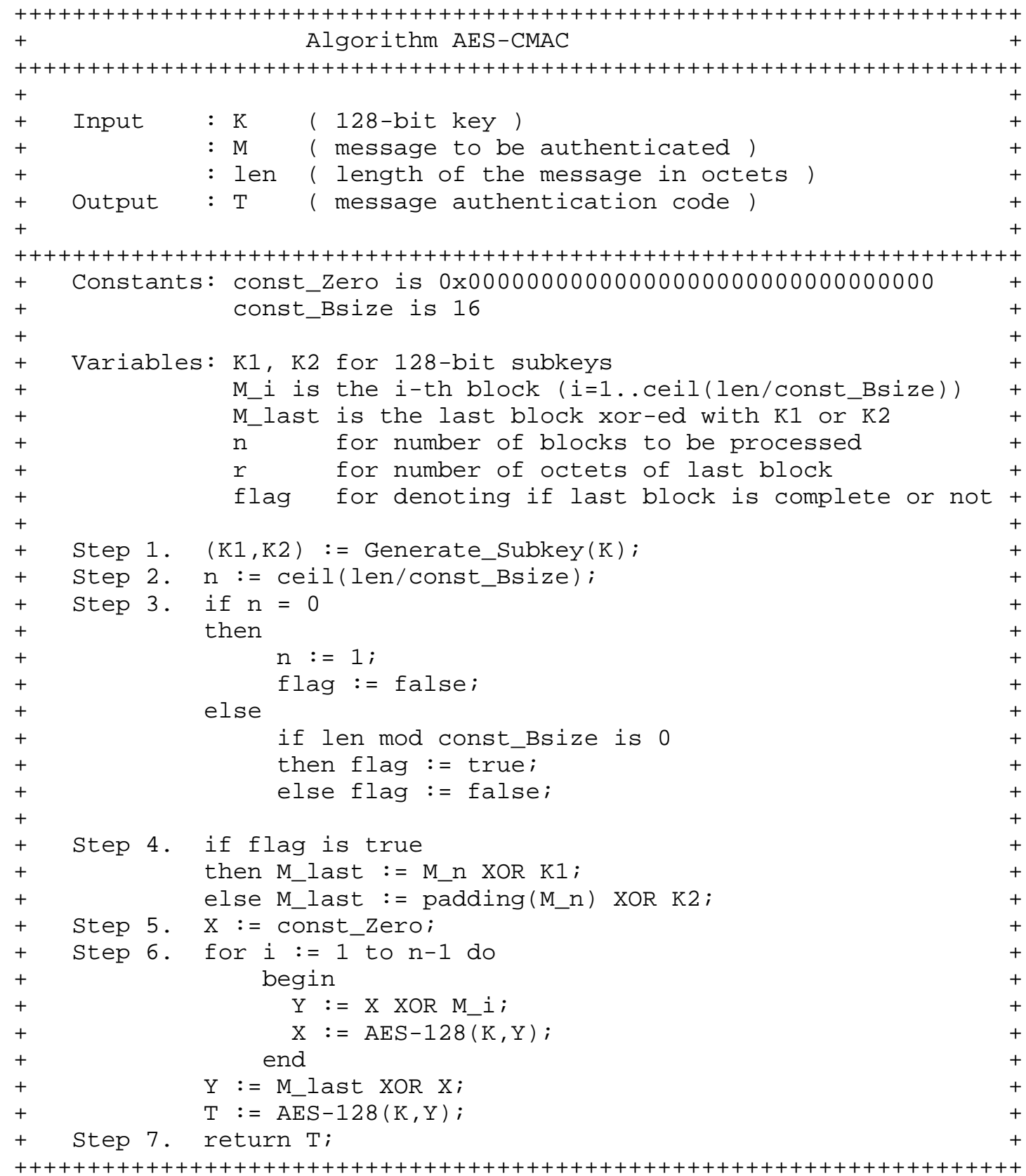

Figure 2.3. Algorithm AES-CMAC 
In step 1, subkeys $\mathrm{K} 1$ and $\mathrm{K} 2$ are derived from $\mathrm{K}$ through the subkey generation algorithm.

In step 2, the number of blocks, $\mathrm{n}$, is calculated. The number of blocks is the smallest integer value greater than or equal to the quotient determined by dividing the length parameter by the block length, 16 octets.

In step 3, the length of the input message is checked. If the input length is 0 (null), the number of blocks to be processed shall be 1 , and the flag shall be marked as not-complete-block (false). Otherwise, if the last block length is 128 bits, the flag is marked as complete-block (true); else mark the flag as not-complete-block (false).

In step 4, M_last is calculated by exclusive-OR'ing M_n and one of the previously calculated subkeys. If the last block is a complete block (true), then M_last is the exclusive-OR of M_n and K1. Otherwise, M_last is the exclusive-OR of padding(M_n) and K2.

In step 5, the variable $\mathrm{X}$ is initialized.

In step 6, the basic CBC-MAC is applied to M_1,.., M_ $\mathrm{n}-1\}$, M_last.

In step 7, the 128-bit MAC, $\mathrm{T}:=\operatorname{AES}-\mathrm{CMAC}(\mathrm{K}, \mathrm{M}, \mathrm{len})$, is returned.

If necessary, the MAC is truncated before it is returned.

\subsection{MAC Verification Algorithm}

The verification of the MAC is simply done by a MAC recomputation. We use the MAC generation algorithm, which is described in section 2.4 .

The MAC verification algorithm, Verify_MAC(), takes four inputs, a secret key, a message, the length of the message in octets, and the received MAC. These are denoted by $K, M$, len, and $T^{\prime}$, respectively. The output of the MAC verification algorithm is either INVALID or VALID.

Figure 2.4 describes the MAC verification algorithm. 


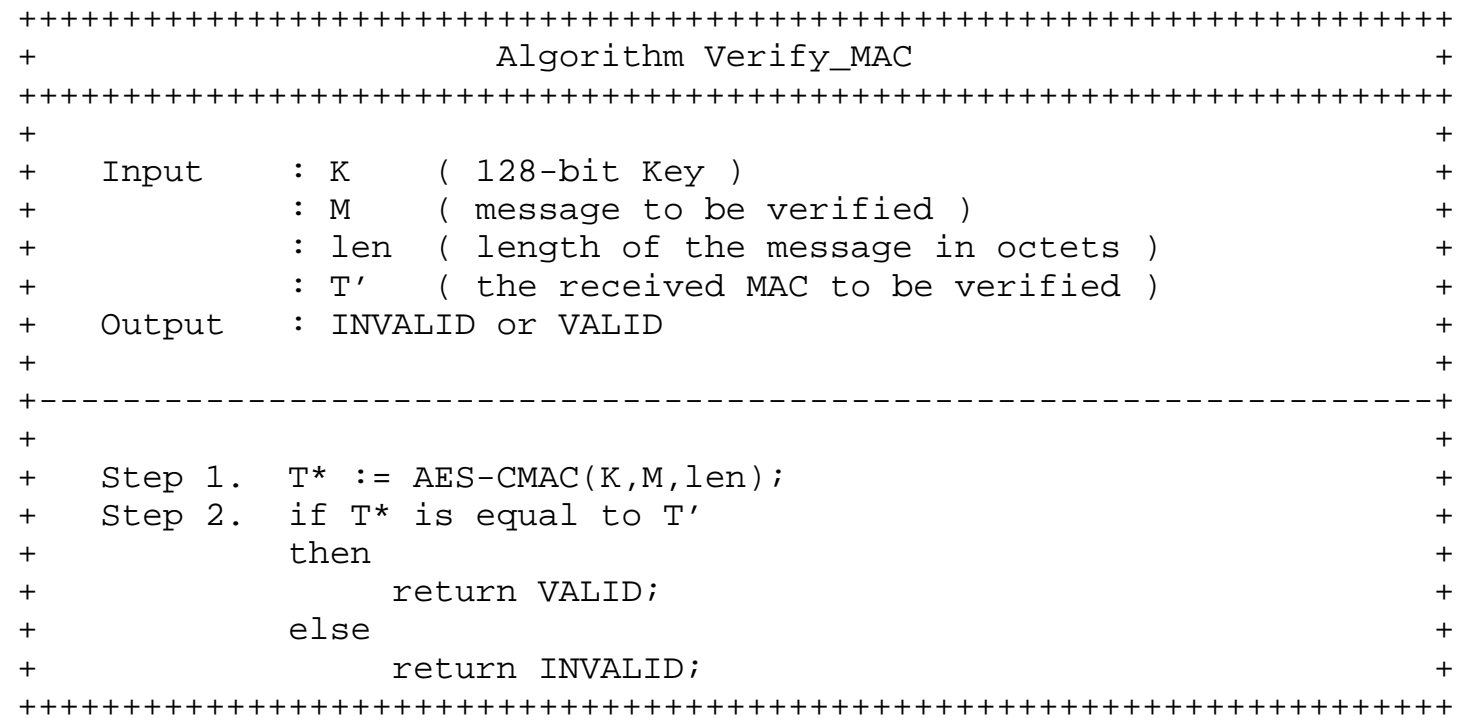

Figure 2.4. Algorithm Verify_MAC

In step $1, T^{*}$ is derived from $K, M$, and len through the MAC generation algorithm.

In step 2, $T^{*}$ and $T^{\prime}$ are compared. If $T^{*}$ is equal to $T^{\prime}$, then return VALID; otherwise return INVALID.

If the output is INVALID, then the message is definitely not authentic, i.e., it did not originate from a source that executed the generation process on the message to produce the purported MAC.

If the output is VALID, then the design of the AES-CMAC provides assurance that the message is authentic and, hence, was not corrupted in transit; however, this assurance, as for any MAC algorithm, is not absolute.

\section{Security Considerations}

The security provided by AES-CMAC is built on the strong cryptographic algorithm AES. However, as is true with any cryptographic algorithm, part of its strength lies in the secret key, $K$, and the correctness of the implementation in all of the participating systems. If the secret key is compromised or inappropriately shared, it guarantees neither authentication nor integrity of message at all. The secret key shall be generated in a way that meets the pseudo randomness requirement of RFC 4086

[RFC4086] and should be kept safe. If and only if AES-CMAC is used 
properly it provides the authentication and integrity that meet the best current practice of message authentication.

4. Test Vectors

The following test vectors are the same as those of [NIST-CMAC]. The following vectors are also the output of the test program in Appendix A.

\begin{tabular}{|c|c|c|c|c|}
\hline \multicolumn{5}{|c|}{ Subkey Generation } \\
\hline K & $2 \mathrm{~b} 7 \mathrm{e} 1516$ & $28 a e d 2 a 6$ & $\mathrm{abf} 71588$ & 09 \\
\hline AES-128 (key, 0) & $7 \mathrm{df} 76 \mathrm{~b} 0 \mathrm{c}$ & $1 \mathrm{ab} 899 \mathrm{~b} 3$ & $3 e 42 f 047$ & b91b546f \\
\hline K1 & fbeed 618 & 35713366 & $7 c 85 e 08 f$ & $7236 a 8$ \\
\hline $\mathrm{K} 2$ & f7ddac30 & $6 a e 266 c c$ & f90bc11e & $e 46 d$ \\
\hline
\end{tabular}

Example 1: len $=0$

M <empty string>

AES-CMAC bb1d6929 e9593728 7 fa37d12 $9 b 756746$

$\begin{array}{lrll}\text { Example 2: len } & =16 \\ \text { M } & 6 \text { bc1bee2 } & \text { 2e409f96 e93d7e11 7393172a } \\ \text { AES-CMAC } & 070 a 16 b 4 & 6 b 4 d 4144 \text { f79bdd9d d04a287c }\end{array}$

--------------------------------------------------

Example 3: len $=40$

M 6bc1bee2 2e409f96 e93d7e11 7393172a

ae2d8a57 1e03ac9c 9eb76fac 45af8e51

$30 \mathrm{c} 81 \mathrm{c} 46$ a35ce 411

AES-CMAC dfa66747 de9ae630 30ca3261 1497 c827

Example 4: len $=64$

M 6bc1bee2 2e409f96 e93d7e11 7393172a

ae2d8a57 1e03ac9c 9eb76fac 45af8e51

$30 \mathrm{c} 81 \mathrm{c} 46$ a35ce411 e5fbc119 1a0a52ef

f69f2445 df4f9b17 ad2b417b e66c3710

AES-CMAC 51fobebf 7e3b9d92 fc497417 79363cfe 


\section{Acknowledgement}

Portions of the text herein are borrowed from [NIST-CMAC]. We appreciate the OMAC1 authors, the SP 800-38B author, and Russ Housley for his useful comments and guidance, which have been incorporated herein. We also thank Alfred Hoenes for many useful comments. This memo was prepared while Tetsu Iwata was at Ibaraki University, Japan.

We acknowledge the support from the following grants: Collaborative Technology Alliance (CTA) from US Army Research Laboratory, DAAD1901-2-0011; Presidential Award from Army Research Office, W911NF-051-0491; NSF CAREER ANI-0093187. Results do not reflect any position of the funding agencies.

6. References

6.1. Normative References

[NIST-CMAC] NIST, Special Publication 800-38B, "Recommendation for Block Cipher Modes of Operation: The CMAC Mode for Authentication", May 2005.

[NIST-AES] NIST, FIPS 197, "Advanced Encryption Standard (AES)", November 2001 .

http://csrc.nist.gov/publications/fips/fips197/fips$197 \cdot \mathrm{pdf}$

[RFC4086] Eastlake, D., 3rd, Schiller, J., and S. Crocker, "Randomness Requirements for Security", BCP 106, RFC 4086 , June 2005 .

6.2. Informative References

[RFC-HMAC] Krawczyk, H., Bellare, M., and R. Canetti, "HMAC: Keyed-Hashing for Message Authentication", RFC 2104, February 1997 .

[OMACla] Tetsu Iwata and Kaoru Kurosawa, "OMAC: One-Key CBC MAC", Fast Software Encryption, FSE 2003, LNCS 2887, pp. 129153, Springer-Verlag, 2003.

[OMAC1b] Tetsu Iwata and Kaoru Kurosawa, "OMAC: One-Key CBC MAC", Submission to NIST, December 2002. Available from the NIST modes of operation web site at http://csrc.nist.gov/CryptoToolkit/modes/proposedmodes / omac/omac-spec.pdf 
[XCBCa] John Black and Phillip Rogaway, "A Suggestion for Handling Arbitrary-Length Messages with the CBC MAC", NIST Second Modes of Operation Workshop, August 2001. Available from the NIST modes of operation web site at http://csrc.nist.gov/CryptoToolkit/modes/proposedmodes / $\mathrm{xcbc}-\mathrm{mac} / \mathrm{xcbc}-\mathrm{mac}-\mathrm{spec} \cdot \mathrm{pdf}$

[XCBCb] John Black and Phillip Rogaway, "CBC MACs for ArbitraryLength Messages: The Three-Key Constructions", Journal of Cryptology, Vol. 18, No. 2, pp. 111-132, Springer-Verlag, Spring 2005. 


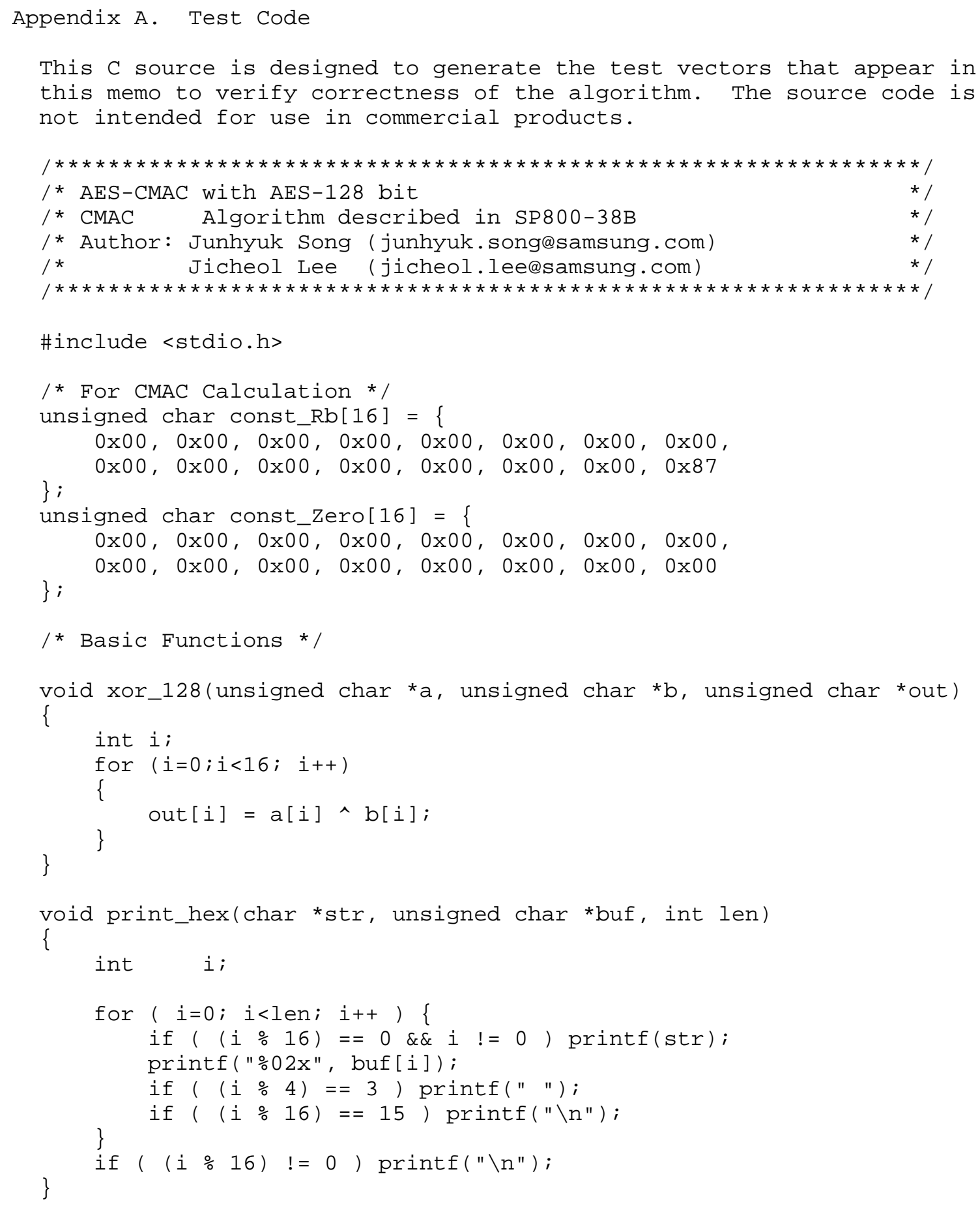




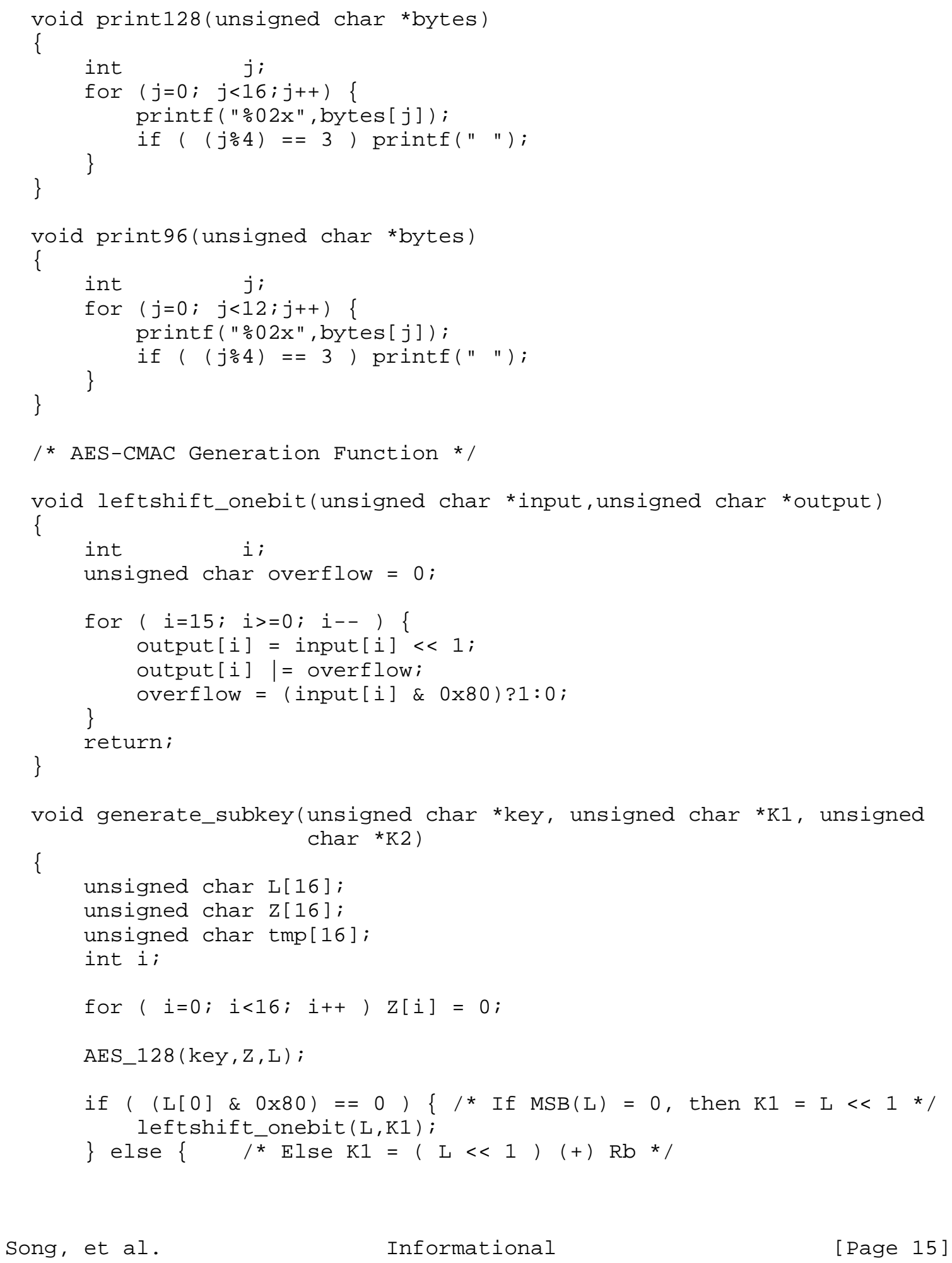




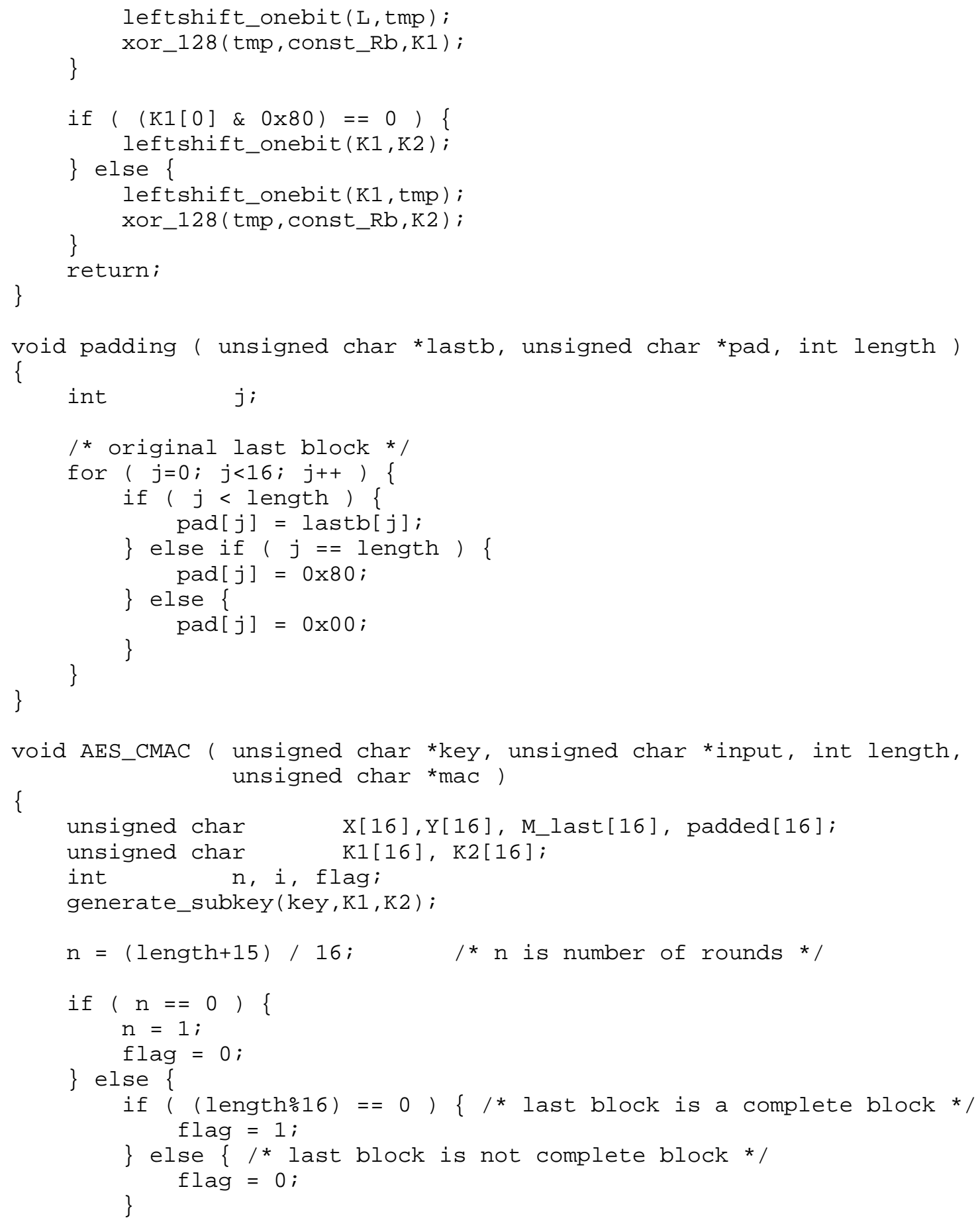




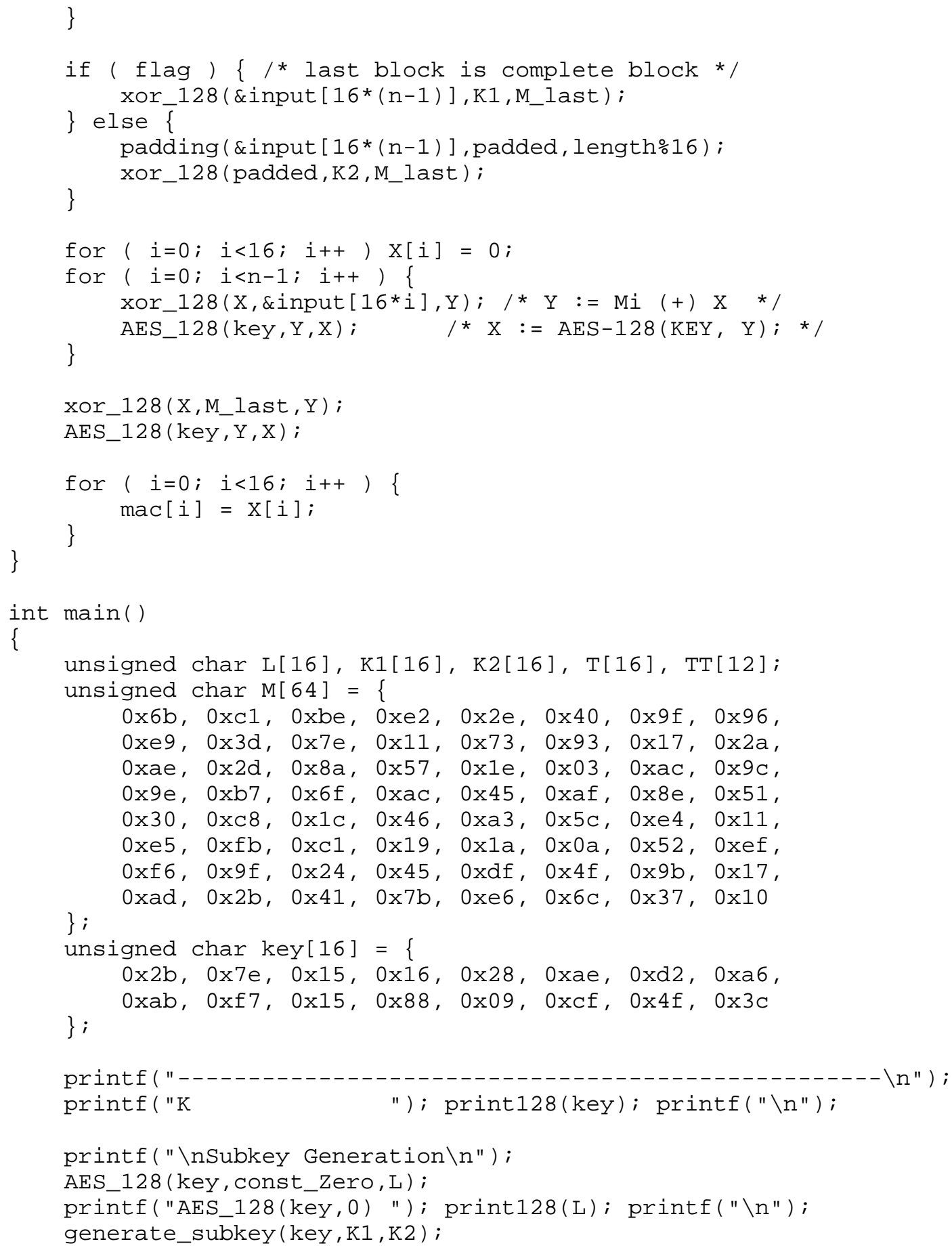




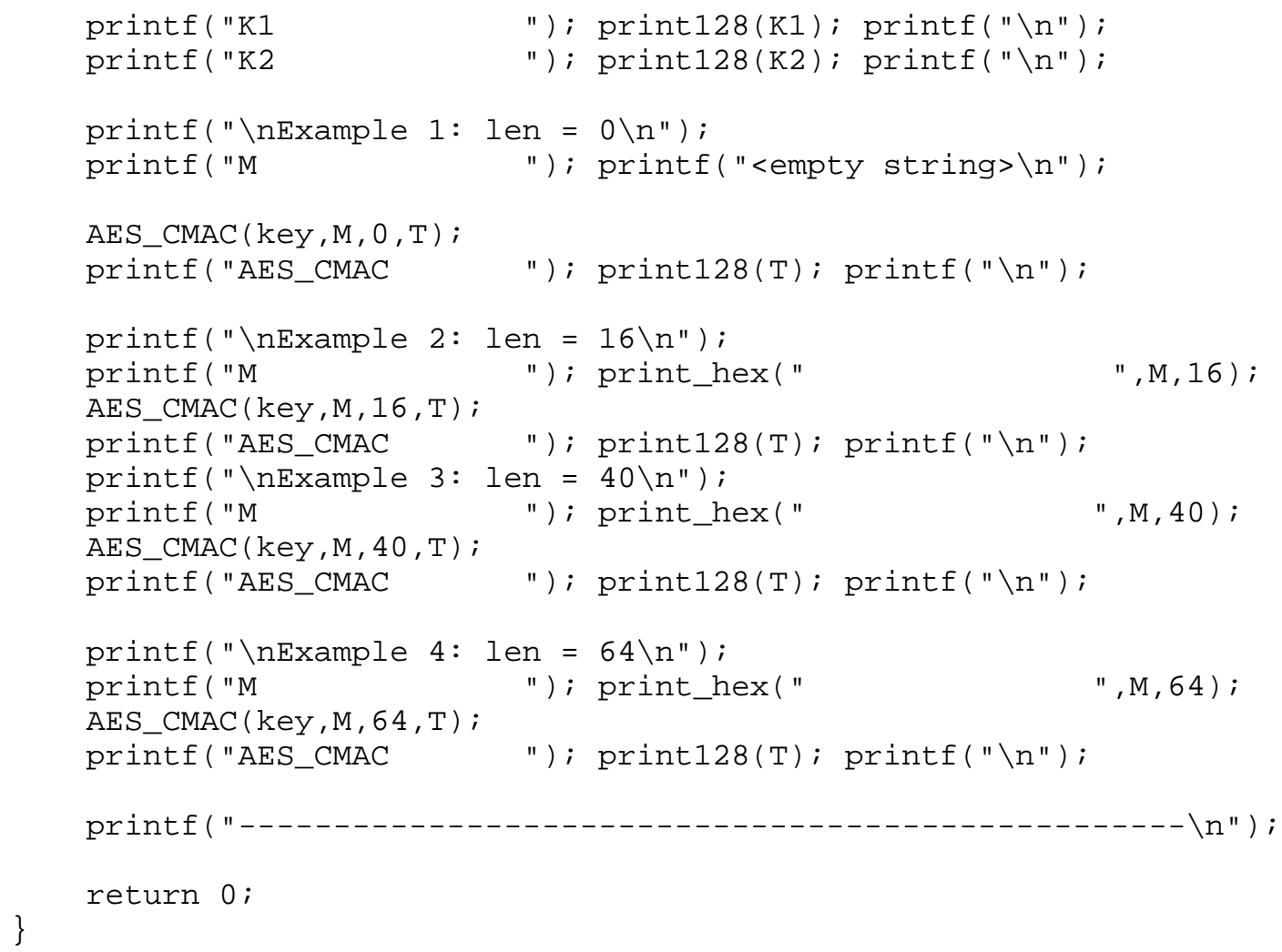




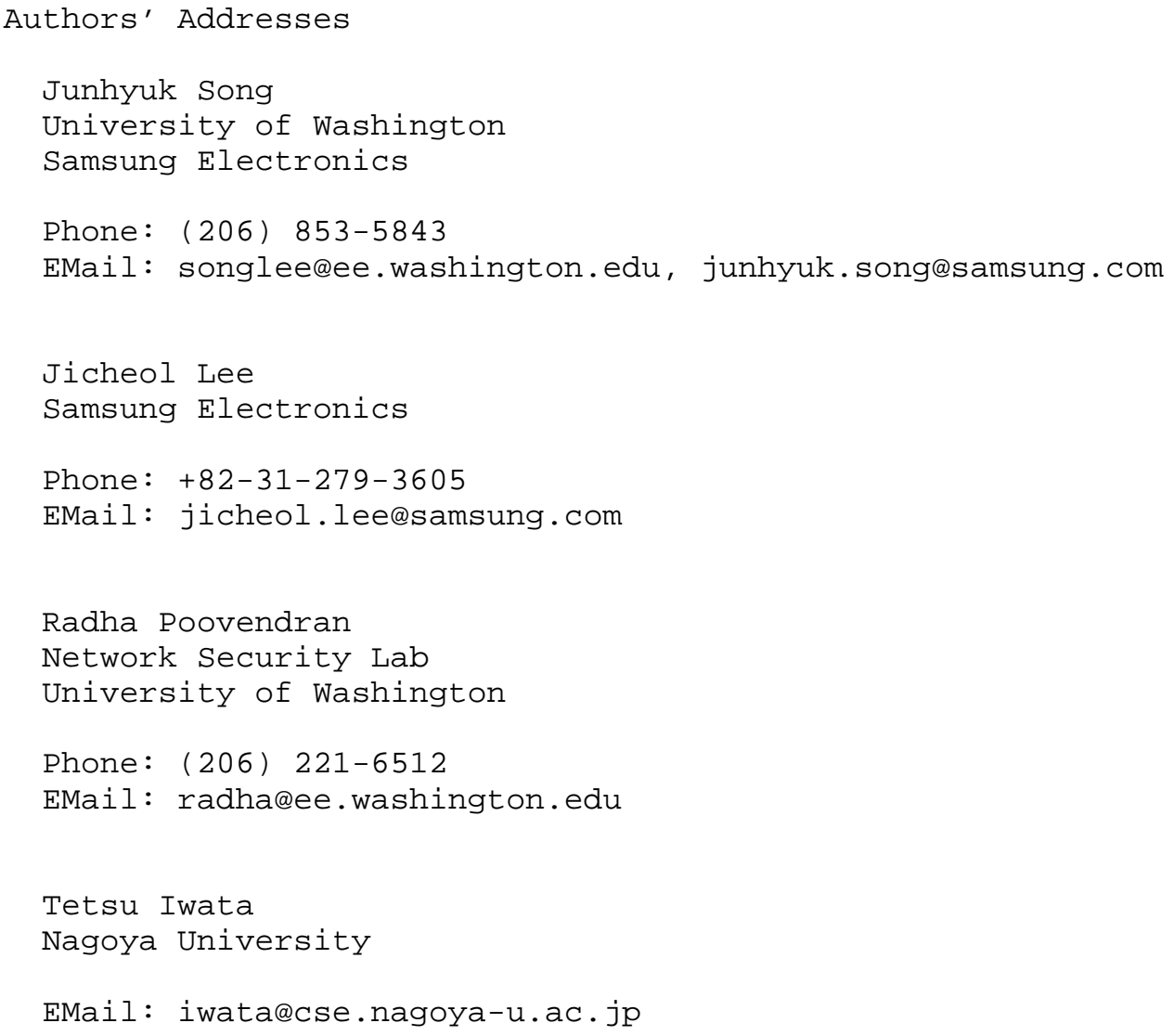


Full Copyright statement

Copyright (C) The Internet Society (2006).

This document is subject to the rights, licenses and restrictions contained in BCP 78, and except as set forth therein, the authors retain all their rights.

This document and the information contained herein are provided on an "AS IS" basis and THE CONTRIBUTOR, THE ORGANIZATION HE/SHE REPRESENTS OR IS SPONSORED BY (IF ANY), THE INTERNET SOCIETY AND THE INTERNET ENGINEERING TASK FORCE DISCLAIM ALL WARRANTIES, EXPRESS OR IMPLIED, INCLUDING BUT NOT LIMITED TO ANY WARRANTY THAT THE USE OF THE INFORMATION HEREIN WILL NOT INFRINGE ANY RIGHTS OR ANY IMPLIED WARRANTIES OF MERCHANTABILITY OR FITNESS FOR A PARTICULAR PURPOSE.

Intellectual Property

The IETF takes no position regarding the validity or scope of any Intellectual Property Rights or other rights that might be claimed to pertain to the implementation or use of the technology described in this document or the extent to which any license under such rights might or might not be available; nor does it represent that it has made any independent effort to identify any such rights. Information on the procedures with respect to rights in RFC documents can be found in BCP 78 and BCP 79 .

Copies of IPR disclosures made to the IETF Secretariat and any assurances of licenses to be made available, or the result of an attempt made to obtain a general license or permission for the use of such proprietary rights by implementers or users of this specification can be obtained from the IETF on-line IPR repository at http://www.ietf.org/ipr.

The IETF invites any interested party to bring to its attention any copyrights, patents or patent applications, or other proprietary rights that may cover technology that may be required to implement this standard. Please address the information to the IETF at ietf-ipreietf.org.

Acknowledgement

Funding for the RFC Editor function is provided by the IETF Administrative Support Activity (IASA). 